\title{
Analysis of the boundary conditions for pyramidal spine fins in extended surface heat transfer
}

\author{
R. G. Carranza ${ }^{1}$ \& J. Ospina ${ }^{2}$ \\ ${ }^{1}$ Chemical Engineering Consultant, Texas, USA \\ ${ }^{2}$ School of Sciences and Humanities, EAFIT University, Colombia
}

\begin{abstract}
Many studies are conducted for pyramidal spine fins relating to temperature profiles and fin efficiencies. However, it is found that a deeper look into the boundary conditions is required. The conditions at the base are simple enough, but the conditions at the tip are more complicated. Despite what condition is applied to the tip, the value at the tip actually has a specific value that is a function of the square root of the Biot number. This result is proven using basic principles from calculus, like the Limit and L'Hospital's rule.

Keywords: heat, transfer, exchanger, fin, efficiency, surface, area, Biot, spine, conduction, convection.
\end{abstract}

\section{Introduction}

The subject in question is the boundary conditions for a pyramidal spine fin. Studies are conducted that investigate the temperature profiles and fin efficiencies relating to a spine fin - for both triangular and square cross-sectional areas [1-5]. During the course of these investigations, a peculiar phenomenon is noticed with respect to the temperature profile of the spine: the analytical solution is the same regardless of the boundary condition defined at the spine tip. This is confirmed using professional computational software, MAPLE; and also by finite differences numerical analysis via fortran software that is written from scratch. The intent here is to prove, using calculus and differential equations, that the temperature profile of a spine fin is uniquely a function of the square root of the Biot number; and is not dependent on the boundary condition specified at the spine tip. 


\section{Spine fin geometry}

Several different types of spine geometries are studied in the literature, as already stated; however, in this work the equilateral triangular cross-sectional area is studied for simplicity. The geometry is presented in fig. 1 and fig. 2.
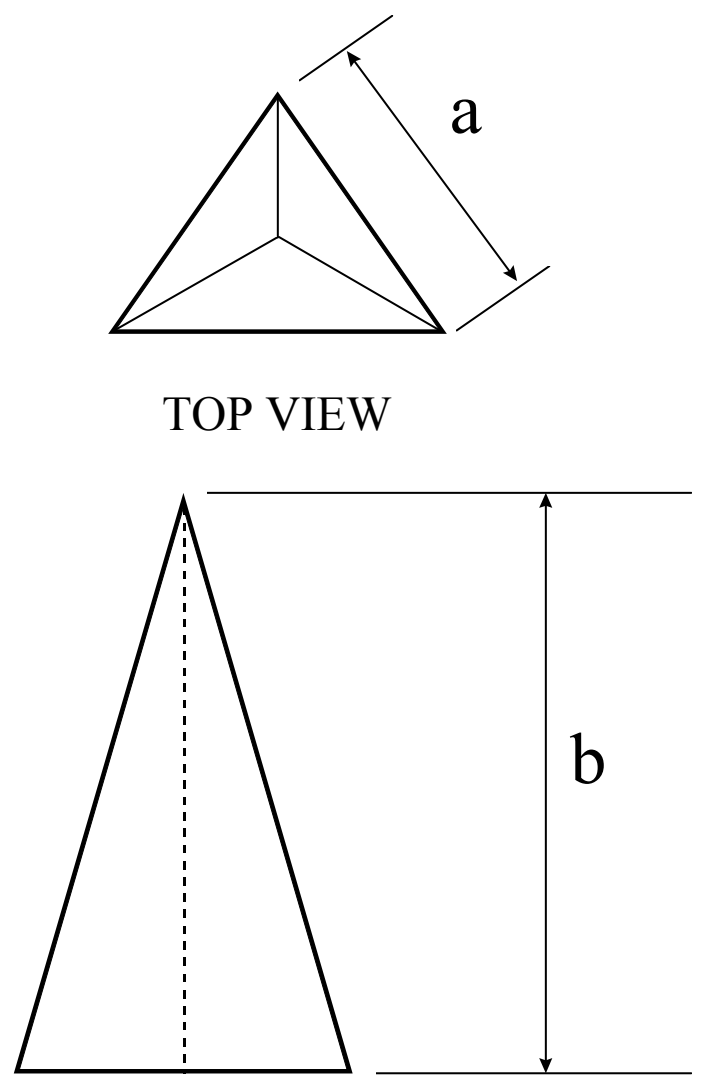

\section{SIDE VIEW}

Figure 1: Schematic of spine geometrical parameters.

Thus, the necessary equations describing the geometry are developed. The geometrical equations are then used to solve the energy balance equation. To start, the width of each spine side, 1; the perimeter, P; and the cross-sectional area, $\mathrm{A}_{\mathrm{c}}$; are all defined as linear or quadratic functions that decrease as they approach the spine tip: 


$$
\begin{gathered}
l(x)=a-\frac{a}{b} x=a-\beta x \\
P=3 l(x)=\frac{d A_{s}}{d x}=3(a-\beta x) \\
A_{c}=\frac{l^{2} \sqrt{3}}{4} \\
A_{c}(x)=\frac{\sqrt{3}}{4}\left(a^{2}-2 a \beta x+\beta^{2} x^{2}\right) \\
\frac{d A_{c}}{d x}=\frac{\sqrt{3}}{2}\left(-a \beta+\beta^{2} x\right)
\end{gathered}
$$

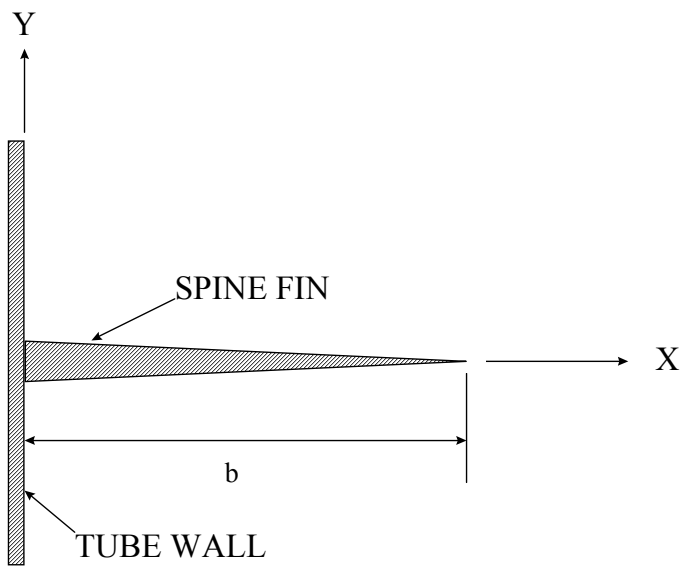

Figure 2: Spatial Coordinate system used in model development.

\section{Spine fin temperature profile: fixed tip temperature condition}

The spine fin temperature profile is determined by conducting an energy balance around the fin. The boundary condition at the tip of the fin is assumed to be a fixed temperature condition. Boundary conditions for the spine fin are $\mathrm{T}(0)=\mathrm{T}_{\mathrm{b}}$ and $\mathrm{T}(\mathrm{b})=\mathrm{T}_{\infty}$. A temperature profile is then determined by using the energy balance, as given in eqn. (6), along with eqns (1)-(5).

$$
\frac{A_{c} d^{2} T}{d x^{2}}+\frac{d A_{c}}{d x} \frac{d T}{d x}-\frac{h_{o}}{k_{m}} \frac{d A_{s}}{d x}\left(T(x)-T_{\infty}\right)=0
$$


It is customary in heat transfer studies to use dimensionless variables. For this reason, eqn (6) is transformed into eqn (7), with the help of eqns (8)-(9) note the following boundary conditions: $\Theta(0)=1$ and $\Theta(1)=0$.

$$
\begin{gathered}
(\mathrm{X}-1) \frac{d^{2} \Theta}{d \mathrm{X}^{2}}+2 \frac{d \Theta}{d \mathrm{X}}+\alpha \Theta=0 \\
\Theta=\frac{T-T_{\infty}}{T_{b}-T_{\infty}} \\
\mathrm{X}=\frac{x}{b}
\end{gathered}
$$

The solution to eqn (7) is not directly attainable. Therefore, it is transformed into a Bessel's equation, with the help of eqns (10)-(12). The Bessel's equation is presented in eqn (13).

$$
\begin{gathered}
\Theta=\frac{u}{\sqrt{X-1}} \\
\frac{d^{2} u}{d X^{2}} \sqrt{X-1}+\frac{d u}{d X} \frac{1}{\sqrt{X-1}}+\frac{(-1+4 \alpha X-4 \alpha) u}{4 \sqrt{(X-1)^{3}}}=0 \\
X=1+\frac{y^{2}}{4 \alpha} \\
\frac{d^{2} u}{d y^{2}}+\frac{1}{y} \frac{d u}{d y}+\left(1-\frac{1}{y^{2}}\right) u=0
\end{gathered}
$$

The solution to eqn (13) is given in eqn (14). If $\Theta(1)=0$, then $\mathrm{C}_{2}$ must equal 0 since $Y_{1}(0)=$ infinity. Applying the second boundary condition, $\Theta(0)=1, C_{1}$ is defined. The results are given in eqns (14)-(17), where eqn (16) is the final form of the equation that describes the temperature profile throughout the length of the fin - note that the results of eqn (16) are also plotted in fig 3.

$$
\Theta=C_{1} \frac{J_{1}(2 \sqrt{\alpha} \sqrt{X-1})}{\sqrt{X-1}}+C_{2} \frac{Y_{1}(2 \sqrt{\alpha} \sqrt{X-1})}{\sqrt{X-1}}
$$




$$
\begin{gathered}
C_{1}=\frac{1}{I_{1}(2 \sqrt{\alpha})} \\
\Theta=\frac{I_{1}(2 \sqrt{\alpha} \sqrt{1-X})}{I_{1}(2 \sqrt{\alpha}) \sqrt{1-X}} \\
\alpha=4 \sqrt{3} \frac{h_{o} b}{\beta k_{m}}=4 \sqrt{3} \frac{h_{o} b^{2}}{a k_{m}}
\end{gathered}
$$

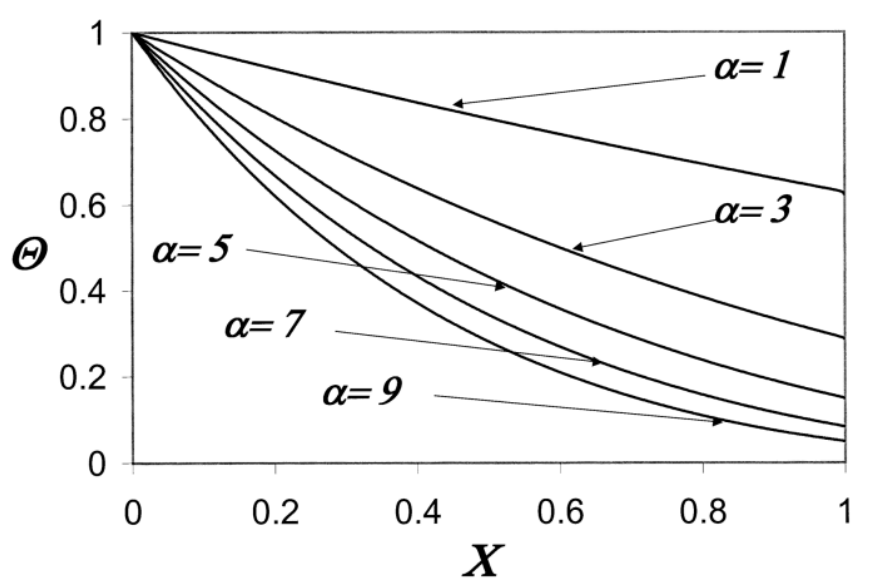

Figure 3: $\Theta$ versus $\mathrm{X}$ for specified values of $\alpha$.

Observe that $\alpha$ is simply the Biot number - the ratio between conduction and convection heat transfer (see Carranza [1] for more detail). Other geometries for spine fins are studied as well, with different types of cross-sectional area: isosceles triangular, scalene triangular, and equilateral square. It is shown that in all 4 cases that temperature profile is described by eqn (16) - the only thing that changes is the definition of $\alpha$. Carranza explains this in more detail [5].

It is now time to focus on eqn (16). Firstly define $u=2 \alpha^{1 / 2}(1-X)^{1 / 2}$. Next, perform the limit on eqn (16) as X approaches 1. L'Hospital's rule must be used in the process. It is clear from eqn (18) that $\Theta$ approaches a definite value as $\mathrm{X}$ approaches 1 .

$$
\begin{aligned}
& \lim _{\mathrm{X} \rightarrow 1} \Theta=\lim _{u \rightarrow 0} \frac{I_{1}(u)}{I_{1}(2 \sqrt{\alpha}) u /(2 \sqrt{\alpha})} \stackrel{\text { L'Hospital }}{\longrightarrow} \\
& \frac{1 / 2\left[I_{0}(0)+I_{2}(0)\right]}{I_{1}(2 \sqrt{\alpha}) /(2 \sqrt{\alpha})}=\frac{\sqrt{\alpha}}{I_{1}(2 \sqrt{\alpha})}
\end{aligned}
$$




\section{Spine fin temperature profile: adiabatic fin tip condition}

For the case of adiabatic fin tip condition, eqn (14) must be examined with the following boundary conditions: $\Theta(0)=1$ and $d \Theta(1) / d X=0$. This time $u=2 \alpha^{1 / 2}$ $(\mathrm{X}-1)^{1 / 2}$. Applying the adiabatic fin tip condition to eqn (14) yields $\mathrm{C}_{2}=0$, since $\mathrm{Y}_{2}(0)$ approaches infinity. Thus, the net result of applying an adiabatic fin tip condition is simply eqns (16) and (18). The details are given in eqns (19) and (20).

$$
\begin{gathered}
\frac{d \Theta}{d \mathrm{X}}=-C_{1} 2 \sqrt{\alpha} u^{-1} J_{2}(u)-C_{2} 2 \sqrt{\alpha} u^{-1} Y_{2}(u) \\
0=C_{1} J_{2}(0)+C_{2} Y_{2}(0)
\end{gathered}
$$

\section{Results and conclusions}

It is proven that the value of $\Theta(1)$ for a pyramidal spine fin, as given in references [1-5], is always a specific value that is a function of the square root of the Biot number:

$$
\Theta(1)=\frac{\sqrt{\alpha}}{I_{1}(2 \sqrt{\alpha})}
$$

Equation 21 is compared to fig. 3 and is also compared to the results obtained from numerical analysis, finite differences. There is no doubt, $\Theta$ equals the value given in eqn 21 when $\mathrm{X}$ equals $1-$ as is proven by calculus and rigorous computation.

\section{Nomenclature}

$\begin{array}{ll}A & \text { area } \\ C & \text { integration constant } \\ I & \text { modified Bessel function } \\ J & \text { Bessel function } \\ P & \text { perimeter } \\ T & \text { temperature } \\ Y & \text { Bessel function of the second kind } \\ a & \text { spine fin side width at base } \\ b & \text { spine fin length } \\ h_{o} & \text { outside individual heat transfer coefficient } \\ k_{m} & \text { thermal conductivity of the fin } \\ l & \text { spine fin side width as a function of } \mathrm{x} \\ u & \text { dummy variable }\end{array}$



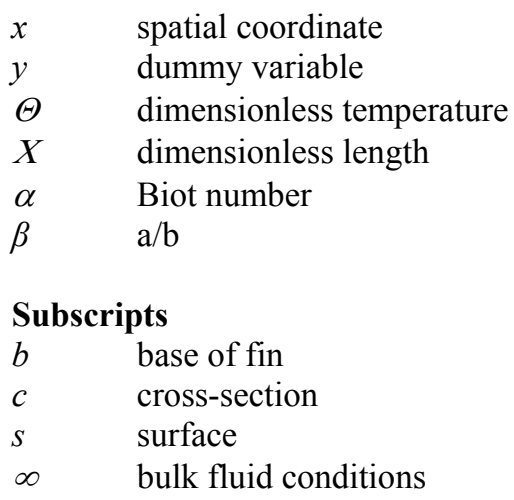

\section{References}

[1] Carranza, R. G., Fin efficiency of a three sided pyramidal spine fin of equilateral triangular and variable cross-sectional area. WSEAS Transactions on Heat and Mass Transfer, 1(1), 2006.

[2] Carranza, R. G., \& Ospina, J., Fin efficiency of a three sided pyramidal spine fin of equilateral triangular and variable cross-sectional area: analytical solution. WSEAS Transactions on Heat and Mass Transfer, 3(1), 2006.

[3] Carranza, R. G., Spine fin efficiency - a three sided pyramidal spine fin of isosceles triangular cross-sectional area. Proc. of the 2007 ASME-JSME Thermal Engineering Summer Heat Transfer Conference, ASME: New York, 2007.

[4] Carranza, R. G., Spine fin efficiency - a three sided pyramidal spine fin of scalene triangular cross-sectional area. Proc. of the $5^{\text {th }}$ WSEAS International Conference on Heat and Mass Transfer, WSEAS: Athens, 2008.

[5] Carranza R. G., Fin efficiency of a four sided pyramidal fin with equilateral square cross-sectional area. Proc. of the $13^{\text {th }}$ UK Heat Transfer Conference, Imperial College: London, 2013. 